\title{
The Evolution of Spiritual Care in the NCCN Distress Management Guidelines
}

\author{
George Handzo, MA, MDivª; Jill M. Bowden, MDiv; and Stephen King, MDiv, PhDc
}

\begin{abstract}
Spiritual care and chaplaincy have been part of the NCCN Clinical Practice Guidelines in Oncology (NCCN Guidelines) for Distress Management since the first meeting of the panel in 1997, possibly the first time this degree of spiritual care and chaplaincy care integration occurred in cancer care. Since that time, the chaplaincy care section of the guidelines, especially chaplain assessment categories derived from a spiritual care assessment, have provided a major resource for healthcare chaplaincy and have served as a model for integrating chaplaincy into the overall team practice of healthcare. However, this section of the NCCN Guidelines has not been substantially updated since it was originally written. During those 20 years, the practice of healthcare chaplaincy and the research that supports it have grown substantially. In the last year, at the request of the panel, we have updated the chaplaincy care section to fully integrate recently published evidence in spiritual care in healthcare, adding more value to this important set of guidelines. Those updates appear in the 2019 version of the NCCN Guidelines. This article discusses the history of chaplaincy involvement in the NCCN Guidelines for Distress Management and the precedent it set for the integration of chaplaincy in other efforts that followed. Integration of this section of the Guidelines into the spiritual care practice at Memorial Sloan Kettering Cancer Center is presented as an example of how these guidelines can be put into practice to improve patient care. Finally, a summary of the recent research by Drs. Kenneth Pargament and Julie Exline is presented as the foundation for the revised chaplain assessment categories and interventions.
\end{abstract}

J Natl Compr Canc Netw 2019;17(10):1257-1261 doi: $10.6004 /$ jnccn.2019.7352

aHealth Services Research \& Quality, HealthCare Chaplaincy Network, New York, New York; ${ }^{b}$ Chaplaincy Services, Memorial Sloan Kettering Cancer Center, New York, New York; and 'Spiritual Health, Child Life and Clinical Navigators, Seattle Cancer Care Alliance, Seattle, Washington.

\section{The History}

NCCN is an alliance of 28 leading cancer centers devoted to patient care, research, and education, begun in $1995 .{ }^{1}$ The NCCN Distress Management Panel was formed in 1997 with Dr. Jimmie Holland as chair, a position she would hold until her death in 2018. Dr. Holland's vision was that the panel should be thoroughly interdisciplinary. Thus, a chaplain was included from the first meeting, along with social workers, psychologists, nurses, oncologists, and psychiatrists. Although it was not unusual even then to have a chaplain serving inpatients with cancer and on staff at cancer centers, integrating a chaplain into a cancer team, and especially into policy and guideline development, was not standard practice as it is today. This integration was especially rare in the outpatient setting, which is where these guidelines were meant to be implemented.

To put this vision in perspective, the first edition of the National Consensus Project Clinical Practice Guidelines for Quality Palliative Care (NCP) did not appear until 2004, and although it attended to the spiritual dimension of care from the beginning, chaplains were not officially integrated into the development of the guidelines until the fourth edition, which was published in $2018 .^{2}$ To qualify to have members officially involved in the guidelines process, an organization had to be a member of the National Coalition for Hospice and Palliative Care. There were no chaplain association members of the coalition until after the publication of the third edition of the NCP guidelines.

The panel chose "distress" as the focus of the 1997 NCCN Guidelines because that term did not represent or infer any kind of pathology. Efforts were made to describe distress as a normally expected reaction to a cancer diagnosis, albeit one that was at least "unpleasant." The term "distress" was chosen because it is more acceptable and less stigmatizing than "psychiatric," "psychosocial," or "emotional"; sounds "normal" and less embarrassing; and can be defined and measured by self-report. The NCCN Guidelines define distress as "a multifactorial unpleasant experience of a psychological (ie, cognitive, behavioral, emotional), social, spiritual, and/or physical nature that may interfere with the ability to cope effectively with cancer, its physical symptoms, and its treatment. 
Distress extends along a continuum, ranging from common normal feelings of vulnerability, sadness, and fears to problems that can become disabling, such as depression, anxiety, panic, social isolation, and existential and spiritual crisis." ${ }^{3}$

The NCCN Guidelines recommend patients be screened for distress at every visit or at regular intervals. Screening should include psychological/psychiatric, social, and spiritual dimensions of distress. Clinicians are then provided guidance on when to make referrals to psychologists/ psychiatrists, social workers, and/or chaplains (see DIS-4 in the NCCN Guidelines for Distress Management, in this issue and at NCCN.org). It is critical for clinicians to understand that these are "guidelines" formulated to "guide" practice, as opposed to treatment protocols meant to be followed explicitly. The decision to make distress the focus of the guidelines was critical to the successful integration of chaplaincy and spiritual care. Then and now, chaplaincy practice specifically excluded the direct treatment of psychopathology. ${ }^{4}$ Using a term such as "depressed" in a chaplain note would be considered outside a chaplain's scope of practice. On the other hand, spiritual distress could easily be understood as a component of chaplaincy practice and an anticipated result for individuals with cancer and their caregivers. ${ }^{5}$

Despite the panel's effort to not focus the guidelines on pathology, it did seek to align with routine medical team practice in which a patient may be screened for issues that need to be attended to immediately, and then thoroughly assessed across all domains. That assessment (usually a formal history and physical) can result in referrals to specialists for in-depth evaluation and diagnosis. Thus, a central feature of the guidelines was the inclusion of a list of chaplaincy assessment issues, such as concerns with meaning and purpose or with one's relationship to the sacred. This alignment established professional chaplains as the spiritual care specialists on the team.

As noted, chaplains have traditionally avoided having any part of their practice "pathologized." Professional chaplains see their work as reinforcing positive spiritual coping or helping patients minimize or overcome spiritual/ religious issues that were impediments to good coping. Chaplains have long resisted any characterization of their work that even appears to reduce a patient's issues to a diagnosis. ${ }^{6,7}$ Thus, when these guidelines were first formulated, it was not surprising that there were no established lists of spiritual assessment categories that would allow the chaplaincy section to parallel the others. Nor was there much evidence to indicate what should be included in such a list. The list created at that time was formulated based on the opinion of a few subject-matter experts. ${ }^{8}$ Today, the evidence for spiritual issues and care has increased exponentially. ${ }^{9-11}$ Thus, the chaplaincy care section is now based on solid research in this domain. That said, much research remains to be performed in areas such as development and validation of quality measures, and development and validation of spiritual assessment tools. ${ }^{12}$

The inclusion of chaplains and particular spiritual concerns in this kind of interdisciplinary enterprise necessitated a panel discussion regarding what issues belong in the spiritual domain of care and what issues belong in the mental health and social work domains of care. This discussion informed the definition of the chaplaincy assessment issues and an effort to use language that would be understood as spiritual rather than psychologic or social. Fundamentally, the difference in scope of practice between the chaplain and the social worker roles is that the chaplain is the spiritual care specialist and emotional care generalist, whereas the social worker is the emotional care specialist and spiritual care generalist. In the end, the only term that appeared on more than one list was "grief" because it was understood to have either a spiritual/existential and/or an emotional/ psychologic etiology.

\section{Use of the Chaplaincy Care Guidelines:}

\section{A Case Example}

The example below is an illustration of how these guidelines might be used by spiritual care providers to improve service delivery. As stated earlier, these are guidelines not practice protocols. Furthermore, the assessment categories developed in 1997 were used because new categories were published too recently to have been trialed. However, there is no reason to believe that the process and outcomes achieved in this example cannot be achieved using the 2019 categories.

Many chaplains structure their clinical time around room-to-room rounds, introducing the religious/spiritual services available in their institutions to new patients and assessing the need for ongoing spiritual care. In addition to this model being inefficient and focused on the patient's desire for a visit rather than spiritual need, it does not translate well to the outpatient setting. Screening for spiritual distress can be performed by any professional provider in a hospital or an outpatient setting. Many studies using various formats have tried to persuade medical professionals to include indicators for the presence of or the tendency toward spiritual struggle, distress, or crises of faith, with varying degrees of success. ${ }^{13}$ In 2009, George Fitchett and James Risk published a screening protocol for spiritual struggle, now known as the Rush Protocol, that is still used in many healthcare organizations. ${ }^{14}$ Spiritual struggle is defined in the protocol as the condition in which religion/spirituality are important to patients but they do not feel they are receiving the strength and comfort from religion/ spirituality that they need. 
In 2013, Memorial Sloan Kettering Cancer Center (MSKCC) had an opportunity to include spiritual screening in the adult inpatient electronic nursing assessment as the programming was being redesigned. Nursing administration accepted a proposal to include the Rush Protocol as part of the revision. Use of the distress thermometer was discussed, but it did not fit the general screening instrument used at the time. Outpatient and pediatric versions were added later.

Variations of the original Rush algorithm are in use in numerous institutions, normally applied in consultation with nursing administration. Guidance is usually included regarding which screening results qualify for a chaplain referral. Some formulations combine the 2 points of the protocol into 1 question; these are a result of negotiation with our medical colleagues and align formats and language with the culture of a given institution, such as changing "chaplain" to "spiritual care provider," which tend to maximize use and outcomes. At MSKCC, consultation with George Fitchett over time has ensured that adherence to the vision and purpose remains consistent.

Embedding the Rush Protocol screening questions in the nursing assessment at MSKCC has dramatically increased the number of referrals for chaplain care throughout the institution and has led to an expansion of spiritual care services into the outpatient clinics and ambulatory care centers.

The exponential increase in referrals for chaplain care has driven documented spiritual care plans that are consistent and measurable and have evidencebased outcomes. To standardize and add value to the plans, key words derived from the spiritual care assessment categories in the original NCCN Guidelines were made a part of the chaplain's evaluation of referrals. This integration and standardization have enabled more actionable feedback to other disciplines on the team, and documentation of the range of spiritual issues present in the patient population. This overall process parallels and mimics the screening, referral, and treatment process envisioned in the NCCN Guidelines for Distress Management. A chart depicting the current findings is shown in Figure 1.

\section{Newly Integrated Revisions to the Chaplaincy Section of the NCCN Guidelines}

Recent revisions to the Chaplaincy Care section in the NCCN Guidelines for Distress Management attempt to include the most current evidence in the field. They seek to capture psycho-religious-spiritual-existential distress. The most referenced research on religious/spiritual distress comes from the work on negative religious coping by Kenneth Pargament and other researchers using his theory of religious coping. His work moved us beyond

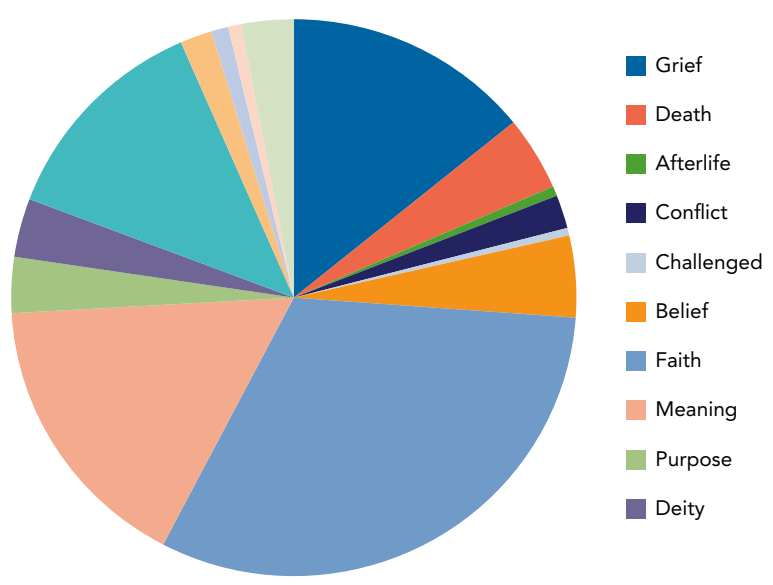

\begin{tabular}{|l|r|r|c|}
\hline & $\begin{array}{c}\text { Visits } \\
\mathbf{n}\end{array}$ & Total & $\begin{array}{c}\text { Unique Patients } \\
\mathbf{n}\end{array}$ \\
\hline Grief & 677 & $18 \%$ & 427 \\
\hline Death & 205 & $5 \%$ & 176 \\
\hline Afterlife & 27 & $1 \%$ & 22 \\
\hline Conflict & 92 & $2 \%$ & 58 \\
\hline Challenged & 21 & $1 \%$ & 20 \\
\hline Belief & 225 & $6 \%$ & 178 \\
\hline Faith & 1,504 & $40 \%$ & 1,086 \\
\hline Meaning & 784 & $21 \%$ & 509 \\
\hline Purpose & 154 & $4 \%$ & 107 \\
\hline Deity & 162 & $4 \%$ & 159 \\
\hline Religious & 606 & $16 \%$ & 520 \\
\hline Religion & 87 & $2 \%$ & 83 \\
\hline Isolation & 47 & $1 \%$ & 410 \\
\hline Guilt & 36 & $1 \%$ & 35 \\
\hline Hopeless & 143 & $4 \%$ & 110 \\
\hline Ritual & 2,156 & $57 \%$ & \multicolumn{1}{c}{$1 \%$} \\
\hline
\end{tabular}

Figure 1. Distribution of chaplaincy diagnoses at Memorial Sloan Kettering Cancer Center.

looking at global indices, such as worship attendance, religious identification, and prayer or attitudes and beliefs, such as intrinsic, extrinsic, and quest orientations, to how one's religion/spirituality functions as one copes with stressors. ${ }^{15}$ This work acknowledges both the benefits provided by religion/spirituality experienced by many people as they cope with stressors ${ }^{16-23}$ (what he called "positive religious coping") and those times when religion/ spirituality is a source of emotional distress and poor coping ("negative religious coping"). ${ }^{11,17-20,24-27}$ Pargament defined negative religious coping as struggles, conflicts, or tensions with what one holds to be sacred, within oneself, and with others regarding spiritual 
matters. ${ }^{15}$ It is this negative religious coping framework that informs considerations of religious/spiritual distress.

Pargament identified 3 dimensions of religious/spiritual distress: transpersonal, interpersonal, and intrapersonal. ${ }^{28,29}$ Transpersonal includes feeling abandoned by, unloved by, punished by (passive), and angry at (active) the sacred/ God/Higher Power/Ground of Being/etc and feeling attacked by evil. Interpersonal includes feeling abused or neglected in one's relationships with regard to spiritual matters. For example, one may feel attacked by or unsupported by one's spiritual community. Intrapersonal includes doubts in one's belief system (eg, I believed that if I was a good person bad things would not happen to me), struggles with morality (eg, questioning whether my medical decisions are aligned with my values), and areas of ultimate meaning (eg, what is the meaning of life).

Pargament's early research used 2 instruments to measure spiritual and religious struggle: $\mathrm{RCOPE}^{30}$ and the Brief RCOPE. ${ }^{15} \mathrm{~A}$ criticism of these instruments was that they were transpersonal-centric. ${ }^{31}$ Recognizing this limitation, in 2014 Pargament, Julie Exline, and others developed a new instrument that is much more inclusive of all 3 dimensions - the Religious and Spiritual Struggles Scale. It has 26 items across 6 domains: the sacred, evil, interpersonal, ultimate meaning, moral, and doubt. ${ }^{31}$ This body of research informed the following items in the Chaplaincy Care algorithm in the NCCN Guidelines: interpersonal conflict regarding spiritual/religious beliefs and practices, concerns with lack of meaning/ purpose, struggles with morality/values, doubts about beliefs, perception of being attacked by evil, and concerns about relationship with the sacred. We further identified conflict between religious beliefs and recommended treatment, ${ }^{32}$ which is not an intrapersonal conflict concerning morals/values but rather between a patient's beliefs and recommended treatment, perhaps representing a different type of interpersonal conflict.

The 2014 research by Exline et $\mathrm{al}^{31}$ also expanded the scale to include more diversity of spirituality, including individuals who identify as religious, religious and spiritual, and spiritual. Many patients diagnosed with cancer identify as neither religious nor spiritual but clearly have existential if not religious and/or spiritual issues. For these patients, the revised guidelines include existential items representing themes found in the Existential Distress Scale (feelings of worthlessness and loneliness $^{33}$ ) and Existential Concerns Questionnaire (concerns about dying/death and/or afterlife ${ }^{34}$ ). Clearly these additional 3 items are concerns that could be experienced by anyone regardless of religious and/or spiritual identity. (Note that there is some overlap between the existential concerns and the concerns in Exline

\begin{tabular}{|l|l|}
\hline $\begin{array}{l}\text { Table 1. Interventions to Address Psycho- } \\
\text { Religious-Spiritual-Existential Concerns }\end{array}$ \\
\hline Intervention & Examples \\
\hline Support/Care & Companioning, listening, nurturing \\
\hline Counseling & $\begin{array}{l}\text { Depth exploration, spiritual journey, legacy } \\
\text { work, spiritual journey work, ethical } \\
\text { consultation }\end{array}$ \\
\hline Education & $\begin{array}{l}\text { Psycho-spiritual or religious teaching, } \\
\text { critical reflection, providing resources like } \\
\text { books or articles }\end{array}$ \\
\hline Ritual & $\begin{array}{l}\text { Communion, anointing, confession by the } \\
\text { patient or by the religious representative } \\
\text { on behalf of an offending body, absolution }\end{array}$ \\
\hline Meditation/Prayer & $\begin{array}{l}\text { Local faith community representatives, } \\
\text { pastoral psychotherapists, spiritual } \\
\text { directors, etc. }\end{array}$ \\
\hline $\begin{array}{l}\text { Referral to community } \\
\text { resources }\end{array}$ & $\begin{array}{l}\text { Social work, psychiatry, palliative care, etc. } \\
\text { Referral to internal resources }\end{array}$ \\
\hline
\end{tabular}

et $\mathrm{al}^{31}{ }^{31}$ such as regarding meaning/purpose and morality/guilt.)

Finally, the 10 items mentioned do not necessarily capture the losses and grief potentially associated with each of them or other concerns. Patients with cancer experience vast and diverse amounts of potential and actual losses/grief, such as loss of health, innocence, normalcy, and relationships, and the consequent grief. Therefore, grief/loss ${ }^{35}$ was included as the eleventh item in the psycho-spiritual-religious-existential distress assessment. Ritual needs was added to accommodate those who wanted a ritual but had no other spiritual issues.

\section{Interventions}

A list of specific interventions to address these psychoreligious-spiritual-existential concerns could be lengthy. For example, from the chaplaincy taxonomy developed by Massey et al alone, ${ }^{36}$ a number of interventions can be identified, such as facilitate communication, facilitate life review, facilitate preparing for end of life, perform a religious rite or ritual, pray, provide grief resources, and provide spiritual/religious resources. The revised list provides more general types of interventions to make it more manageable (Table 1). Most of this intervention nomenclature has been adapted from Spiritual Health Victoria's Spiritual Care Minimum Data Set Framework. ${ }^{37}$

\section{Conclusions}

Involvement of chaplaincy in the NCCN Guidelines for Distress Management and the inclusion of chaplaincy assessment categories were visionary at inception. The NCCN Guidelines remain one of the major sets of guidelines that drive practice in spiritual care in healthcare. 
The screening process at MSKCC is an example of how the guidelines have significantly contributed to the integration of spiritual care in cancer care. The new revisions to the chaplaincy section integrate recent research that supports a new set of assessment categories. Although this is a significant advance for chaplaincy in the oncology sector, much more research is needed. Prime areas for investigation include further validation of spiritual screening tools, the relationship of the various assessment categories to the effectiveness of the interventions proposed, and outcomes, such as patient satisfaction and use of aggressive resources at the end of life.

Submitted June 3, 2019; accepted for publication August 29, 2019.

The authors have disclosed that they have no financial interests, arrangements, or affiliations with the manufacturers of any products discussed in this article or their competitors.

Correspondence: George Handzo, MA, MDiv, 15021 Marble Drive, Sherman Oaks, CA 91403. Email: ghandzo@healthcarechaplaincy.org

\section{References}

1. National Comprehensive Cancer Network. About NCCN. Available at: https://www.nccn.org/about/default.aspx. Accessed August 15, 2019.

2. National Coalition for Hospice and Palliative Care. Clinical Practice Guidelines for Quality Palliative Care, 4th edition. Available at: https:// www.nationalcoalitionhpc.org/ncp/. Accessed May 26, 2019.

3. Riba MB, Donovan KA, Andersen B. NCCN Clinical Practice Guidelines in Oncology: Distress Management. Version 3. 2019. Accessed August 4 , 2019. To view the most recent version, visit NCCN.org

4. Pryser P. The Minister as Diagnostician. Louisville, KY: Westminster John Knox Press; 1976.

5. Mako C, Galek K, Poppito SR. Spiritual pain among patients with advanced cancer in palliative care. J Palliat Med 2006;9:1106-1113.

6. Handzo GF, Cobb M, Holmes C, et al. Outcomes for professional health care chaplaincy: an international call to action. J Health Care Chaplain 2014;20:43-53

7. Kelly E, Swinton J, eds. Re-membering the Soul of Healthcare: Critical Reflections on the Future of Health and Social Care Chaplaincy. London, England: Jessica Kingsley Publishers, in press.

8. NCCN practice guidelines for the management of psychosocial distress. National Comprehensive Cancer Network. Oncology (Williston Park) 1999;13:113-147

9. Steinhauser K, Fitchett G, Handzo G, et al. Spirituality and palliative care research state of the science consensus results part i: definitions, taxonomy, measurement and outcomes. J Pain Symptom Manage 2017; 54:428-440.

10. Astrow AB, Kwok G, Sharma RK, et al. Spiritual needs and perception of quality of care and satisfaction with care in hematology/medical oncology patients: a multicultural assessment. J Pain Symptom Manage 2018;55:56-64.e1.

11. Balboni TA, Vanderwerker LC, Block SD, et al. Religiousness and spiritual support among advanced cancer patients and associations with endof-life treatment preferences and quality of life. J Clin Oncol 2007;25: 555-560.

12. Fitchett G. Recent progress in chaplaincy-related research. J Pastoral Care Counsel 2017;71:163-175.

13. Borneman T, Ferrell B, Puchalski CM. Evaluation of the FICA tool for spiritual assessment. J Pain Symptom Manage 2010;40:163-173.

14. Fitchett G, Risk JL. Screening for spiritual struggle. J Pastoral Care Counsel 2009;63:4-1-12.

15. Pargament K, Feuille M, Burdzy D. The Brief RCOPE: current psychometric status of a short measure of religious coping. Religions (Basel) 2011;2: 51-76.

16. Canada AL, Fitchett G, Murphy PE, et al. Racial/ethnic differences in spiritual well-being among cancer survivors. J Behav Med 2013;36: 441-453.

17. Tarakeshwar N, Vanderwerker LC, Paulk E, et al. Religious coping is associated with the quality of life of patients with advanced cancer. J Palliat Med 2006;9:646-657

18. Boscaglia N, Clarke DM, Jobling TW, et al. The contribution of spirituality and spiritual coping to anxiety and depression in women with a recent diagnosis of gynecological cancer. Int J Gynecol Cancer 2005;15:755-761.
19. Fitchett G, Murphy PE, Kim J, et al. Religious struggle: prevalence, correlates and mental health risks in diabetic, congestive heart failure, and oncology patients. Int J Psychiatry Med 2004;34:179-196.

20. Thuné-Boyle IC, Stygall J, Keshtgar MR, et al. Religious/spiritual coping resources and their relationship with adjustment in patients newly diagnosed with breast cancer in the UK. Psychooncology 2013;22:646-658.

21. Aukst-Margetić $B$, Jakovljević $M$, Margetić $B$, et al. Religiosity, depression and pain in patients with breast cancer. Gen Hosp Psychiatry 2005;27: 250-255.

22. Gall TL, Cornblat MW. Breast cancer survivors give voice: a qualitative analysis of spiritual factors in long-term adjustment. Psychooncology 2002; 11:524-535.

23. Tallman B, Shaw K, Schultz J, et al. Well-being and posttraumatic growth in unrelated donor marrow transplant survivors: a nine-year longitudinal study. Rehabil Psychol 2010;55:204-210.

24. Exline JJ, Prince-Paul M, Root BL, et al. The spiritual struggle of anger toward God: a study with family members of hospice patients. J Palliat Med 2013;16:369-375.

25. Manning-Walsh J. Spiritual struggle: effect on quality of life and life satisfaction in women with breast cancer. J Holist Nurs 2005;23:120-140, discussion 141-144.

26. Exline JJ, Park CL, Smyth JM, et al. Anger toward God: social-cognitive predictors, prevalence, and links with adjustment to bereavement and cancer. J Pers Soc Psychol 2011;100:129-148.

27. Zwingmann $C$, Wirtz M, Müller $C$, et al. Positive and negative religious coping in German breast cancer patients. J Behav Med 2006;29:533-547.

28. Pargament K. The Psychology of Religion and Coping: Theory, Research, Practice. New York, NY:Guilford Press; 1997.

29. Pargament KI. Spiritually Integrated Psychotherapy: Understanding and Addressing the Sacred. New York, NY: Guilford Press; 2007

30. Pargament KI, Koenig HG, Perez LM. The many methods of religious coping: development and initial validation of the RCOPE. J Clin Psychol 2000;56:519-543.

31. Exline JJ, Pargament KI, Grubbs JB, et al. The Religious and Spiritual Struggles Scale: development and initial validation. Psychol Relig Spiritual 2014;6:208-222

32. Piderman KM, Marek DV, Jenkins SM, et al. Patients' expectations of hospital chaplains. Mayo Clin Proc 2008;83:58-65.

33. Lo C, Panday T, Zeppieri J, et al. Preliminary psychometrics of the Existential Distress Scale in patients with advanced cancer. Eur J Cancer Care (Engl) 2017;26:1-8.

34. van Bruggen $V$, Ten Klooster P, Westerhof $G$, et al. The Existential Concerns Questionnaire (ECQ)-development and initial validation of a new existential anxiety scale in a nonclinical and clinical sample. J Clin Psychol 2017;73:1692-1703.

35. Burke L, Neimeyer R. The inventory of complicated spiritual grief: assessing spiritual crisis following loss. Religions (Basel) 2016;7:1-10.

36. Massey K, Barnes MJ, Villines D, et al. What do I do? Developing a taxonomy of chaplaincy activities and interventions for spiritual care in intensive care unit palliative care. BMC Palliat Care 2015;14:10.

37. Spiritual Health Victoria. Spiritual Care Minimum Data Set Framework. Available at: www.spiritualhealthvictoria.org.au/LiteratureRetrieve.aspx? $\mathrm{ID}=207060$. Accessed May 22, 2019. 